\title{
Wound degradomics Current status and future perspectives
}

\section{Review Article}

Author(s):

Hermes, Olivia; Schlage, Pascal; auf dem Keller, Ulrich

Publication date:

2011-11

Permanent link:

https://doi.org/10.3929/ethz-b-000160041

Rights / license:

In Copyright - Non-Commercial Use Permitted

Originally published in:

Biological Chemistry 392(11), https://doi.org/10.1515/BC.2011.092 


\section{Minireview}

\section{Wound degradomics - current status and future perspectives}

\author{
Olivia Hermes, Pascal Schlage and Ulrich auf dem \\ Keller* \\ Institute of Cell Biology, ETH Zurich, 8093 Zurich, \\ Switzerland \\ * Corresponding author \\ e-mail: ulrich.aufdemkeller@ cell.biol.ethz.ch
}

\begin{abstract}
Proteases are pivotal modulators of extracellular matrix components and bioactive proteins at all phases of cutaneous wound healing and thereby essentially contribute to the successful reestablishment of skin integrity upon injury. As a consequence, disturbance of proteolytic activity at the wound site is a major factor in the pathology of chronic wounds. A large body of data acquired in many years of research provide a good understanding of how individual proteases may influence the repair process. The next challenge will be to integrate these findings and to elucidate the complex interactions of proteolytic enzymes, their inhibitors and substrates on a system-wide level. Here, we present novel approaches that might help to achieve this ambitious goal in cutaneous wound healing research.
\end{abstract}

Keywords: protease web; proteolysis; proteomics; skin; wound healing.

\section{Introduction}

Cutaneous wound healing is a highly complex process that requires precise orchestration of multiple systems involving different cell types. In order to reestablish the barrier function of the skin upon injury, a variety of signaling molecules regulate cell behavior, such as activation, survival and apoptosis, differentiation and migration, in a time and space dependent manner. Mainly the interplay of keratinocytes, fibroblasts, inflammatory and endothelial cells eventually leads to wound closure. In a concerted action, these cells are responsible for essential processes like inflammation, angiogenesis, granulation tissue formation and reepithelialization that end in successful wound repair.

The process of wound healing is generally divided into three overlapping phases: inflammation, new tissue formation and remodeling (Gurtner et al., 2008). At the beginning of the first phase, a platelet plug associated with insoluble fibrin fibers is responsible to achieve hemostasis. A provisional matrix consisting of cross-linked fibrin, fibronectin and other extracellular matrix (ECM) proteins is formed in the wound bed and serves as a protective barrier against water loss and invading bacteria. In addition, this matrix becomes a scaffold for fibroblasts and endothelial cells and for infiltrating inflammatory cells, which are attracted to the site of injury by cytokines, chemokines and inflammatory mediators. After a few days, the provisional matrix is disentangled from the wound as an eschar. In the second stage, 2-10 days after skin injury, keratinocytes migrate into the wound and start to proliferate, resulting in wound reepithelialization. The wound clot is gradually replaced by granulation tissue, which is characterized by sprouting blood vessels, the presence of macrophages and other immune cells and fibroblasts that deposit ECM. Fibroblasts are attracted from the undamaged dermis or recruited from the bone marrow and activated by growth factors like platelet-derived growth factor and transforming growth factor $\beta$ that stimulate proliferation and migration. Some fibroblasts differentiate into myofibroblasts, cells responsible for wound contraction. The final stage of wound repair starts about 2 weeks after injury and lasts up to a year or more. During this stage of wound maturation, a collagenous scar is formed, the number of fibroblasts in the wound decreases, and the number of blood vessels is reduced by apoptosis of endothelial cells. Myofibroblasts and macrophages either undergo apoptosis or exit the site of injury. The remaining fibroblasts start to remodel the collagenous dermis and rearrange collagen fibers to optimize tensile strength, which, however, never recovers the stiffness of unwounded skin. In addition, healed skin does not contain skin appendages like hair follicles, sweat glands and sebaceous glands.

Proteases play important roles in all phases of cutaneous wound repair to facilitate invasion of inflammatory cells, migration of fibroblasts and keratinocytes, formation of new blood vessels, wound contraction and finally remodeling of the scar tissue. Thereby, they are not only involved in ECM degradation, but specifically modify bioactive proteins, such as chemokines, cytokines and growth factors. Furthermore, proteases like kinases do not act alone, but are interconnected in a dynamic network that has been termed the 'protease web' (auf dem Keller et al., 2007). Thus, they are now considered as pivotal signaling mediators with central roles in the orchestration of complex processes on the cellular and on the tissue level during skin wound healing. While many studies focused on the analysis of matrix metalloproteinases (MMPs) as classical 'matrix degraders', more recent research started to elucidate MMP activity towards bioactive mediators and their impact on wound inflammation and angiogenesis (Page-McCaw et al., 2007). In addition, the important contributions of members of other protease classes, such as ADAMs (a disintegrin and metalloprotease), astacins (tolloids and meprins), cathepsins, kallikreins, and matriptases, 
to the healing process became a particularly active field of research (Moali and Hulmes, 2009).

In this minireview we will give a short overview of approaches to assess parameters in protease research in cutaneous wound repair with an outlook on how novel technologies may influence the field in the future. Thereby, we will focus on the study of proteases in in vivo and in vitro models of skin repair. For a comprehensive overview of protease biology in wound healing, taking into account additional data from the analysis of proteolytic activity in similar processes, such as inflammation, angiogenesis and carcinogenesis, the reader is referred to two excellent recent reviews by Toriseva and Kähäri (2009) and Moali and Hulmes (2009).

\section{Defining the wound transcriptional degradome}

A wealth of information is available on the mRNA expression of proteases at the wound site (transcriptional degradome) and during the time course of the healing process. In this respect, members of the MMP family have been studied most extensively due to their implication in all phases of cutaneous wound repair. Technically, detailed expression data for most proteases have been acquired using Northern blots, RNase protection assays and in situ hybridizations in human and mouse skin wounds (Toriseva and Kähäri, 2009). Employing these targeted approaches allowed the temporal and spatial assessment of protease transcripts with high specificity. While this is an inherent advantage, the analysis of single transcripts reveals no information about the concomitant expression of other proteases that are either directly or indirectly linked to the protease under study. Addressing this limitation, some studies monitored the expression of multiple proteases of the same or of different classes within the same experiment (Madlener et al., 1998), whereby either predicted transcriptional linkages between the examined protease genes could be verified or new relations established. In addition, the careful selection of samples in human studies and standardized procedures allows meaningful conclusions when comparing expression data for individually assessed proteases from independent studies (Toriseva and Kähäri, 2009).

However, transcripts for all proteases and their quantitative changes at important steps of the repair process should ideally be analyzed in the same experiment. Since this possibility became available with the invention of the cDNA microarray technology, several studies have applied this tool to the analysis of cutaneous wound repair (Deonarine et al., 2007). Although differential expression in wounded versus normal skin could be corroborated for many proteases, some previously validated transcripts might evade detection in microarray analyses. This could be either due to incomplete transcript coverage of the array used or the generally lower sensitivity compared to Northern blotting, RNase protection assay and quantitative real-time (qRT)-PCR. To address the first issue, dedicated microarrays have been developed for the study of proteases, the $\mathrm{Hu} / \mathrm{Mu}$ ProtIn array and the CLIP$\mathrm{CHIP}^{\mathrm{TM}}$. Due to its unique design the $\mathrm{Hu} / \mathrm{Mu}$ ProtIn array allows differential assessment of protease mRNAs from mice and humans in the same sample and thus is particularly suited for xenograft studies in mice (Schwartz et al., 2007). Since this leads to reduced coverage based on the exclusion of species cross-hybridizations, the CLIP-CHIP ${ }^{\mathrm{TM}}$ is the most comprehensive two-color microarray for proteases, their inhibitors and inactive homologues (Kappelhoff et al., 2010). Hence, it provides a valuable and cost-effective alternative to high-density exon arrays for the specific analysis of protease mRNA expression in wound healing. To achieve comprehensive coverage with concomitant high sensitivity, profiling of a complete protease class by qRT-PCR has been proven very useful. Thereby, particular efforts have been dedicated to the development of specific probes for measuring complete sets of MMP, TIMP (tissue inhibitor of matrix metalloproteinases), ADAM and ADAMTS (a disintegrin and metalloprotease with thrombospondin motifs) transcripts in human tumor samples that provide validated tools also for the study of skin repair (Hodgkinson et al., 2010; Pennington and Edwards, 2010). In most cases total RNA for microarray or qRT-PCR analysis was isolated from tissue lysates neglecting any spatial information for the measured transcripts within the tissue. However, the latter is particularly important for the study of processes employing multiple cell types that dynamically invade and evade the tissue, such as cutaneous wound healing (Nanney et al., 2006). An elegant method to gain this information is laser capture microdissection (LCM) that researchers have successfully used to specifically study gene expression in blood vessels from human wound-edge tissue by microarray analysis (Roy et al., 2007) or transcripts of CCN genes during wound healing by qRT-PCR (Rittié et al., 2011).

\section{Exploring the wound translational degradome}

In many studies the analysis of protease transcript levels and locations in the wound were complemented by assessment of expression and cellular distribution of the same proteases on the protein level, providing a good coverage of the translational degradome (Madlener et al., 1998). To acquire these data, mostly Western blot, immunohistochemistry and immunofluorescence techniques were applied, and in some cases protease proteins were quantified in wound fluids or tissue lysates by ELISA (Toriseva and Kähäri, 2009). While highly sensitive and specific, these single protein approaches have the same limitations as similar techniques for the analysis of individual transcripts. With their rapid development in recent years novel mass spectrometry-based proteomics technologies became attractive alternatives for the identification and quantification of proteins in complex proteomes. Of these, both gel-based and gel-free techniques have been applied to the analysis of wound samples (Edsberg, 2009), whereby most studies examined wound fluids that can be easily obtained from patients. In this regard another advantage is the similarity between wound fluid and blood plasma, for which numerous depletion strategies for high abundance proteins, such as albumin, have been developed to facilitate the 
discovery of low abundance proteins, which include many proteases, inhibitors and protease substrates.

In 2008, Fernandez et al. analyzed fluids of chronic human wounds after immunodepletion of highly abundant components by two-dimensional (2D) chromatography and LC-ESI/ MS or MALDI-TOF/TOF. Thereby, they identified about 40 proteins including the protease inhibitor elafin and proteases of the complement system (Fernandez et al., 2008). In a more recent study, Eming et al. compared wound fluids derived from healing and non-healing wounds in a 1D-PAGE LCMS/MS approach and identified among 149 total proteins numerous proteases and inhibitors, such as MMP9, neutrophil elastase, plasminogen, plasma kallikrein, serpins and cystatins (Eming et al., 2010). The most comprehensive list of 1191 proteins has been reported by Escalante et al. in a proteomic analysis of wound fluids of mice wounded by snake venom toxins (Escalante et al., 2009). However, it should be mentioned that wounds in this study were not a result of mechanical injury but of intramuscular injection of toxins. In another study using this model, the same group also analyzed the effect of batimastat, a broad spectrum MMP inhibitor, on protein amounts within wound exudates employing relative quantification by spectral counting. Indeed, they found a batimastat dependent reduction in matrix proteins released into the wound fluid indicating inhibition of MMP activity at the wound site (Rucavado et al., 2011).

However, despite a rapid increase in speed and sensitivity of newly developed mass spectrometers, the comprehensive and reliable identification and quantification of all proteases will most likely be impossible due the overwhelming complexity and dynamic range of protein abundance in wound fluids or tissues. To circumvent this problem a targeted proteomics technique termed selected reaction monitoring (SRM) has been established that allows monitoring of specific proteins in complex proteomes that might be masked in unbiased discovery approaches (Huttenhain et al., 2009). The SRM technology has evolved into a high-throughput technology that enables the simultaneous identification and quantification of hundreds or even thousands of proteins in biological samples. Hence, it is expected that specific SRM assays will soon be available to study the expression of protease classes or even all proteases at important time points during cutaneous tissue repair. Indeed, according to our own searches the SRMAtlas (http://www.mrmatlas.org), a compendium for SRM based targeted proteomics assays (Picotti et al., 2008), currently comprises observed transitions for $66 \%$ of all human and $53 \%$ of all murine proteases annotated in the UniProt/Swiss-Prot database.

Obtaining data on the location of expressed proteins within the heterogeneous wound tissue using mass spectrometrybased proteomics is even more challenging than in transcriptome analyses, particularly since much higher amounts of material are needed that cannot easily be obtained by LCM. However, Wiśniewski et al. recently presented a modified workflow for shotgun proteomics that allowed the identification and quantification of thousands of proteins from microdissected cancer tissues from clinical specimens, dem- onstrating the feasibility of this approach (Wisniewski et al., 2011). Another technique for the spatial analysis of expressed proteases and substrates in wounds is tissue profiling by MALDI MS, where proteins and peptides are detected in tissue slices mounted on a MALDI target (Nanney et al., 2006). Indeed, this technique was successfully used to reveal S100 proteins as being differentially expressed in regenerative versus non-regenerative wounds in mice (Caldwell et al., 2008).

Taken together, these promising studies indicate the potential of proteomics to substantially extend our current knowledge of the wound degradome in time and space at the protein level.

\section{Assessing the wound activity degradome}

Since many proteases are tightly regulated not only on the expression but also on the activity level, the next step in the degradomics analysis of skin repair is the definition of the activity degradome, i.e., all expressed proteases that are active at a given time point after wounding. Gelatin zymography has been widely used to assess the activation status of MMPs in human wound fluids and murine wound lysates. This technique mainly analyzed the activities of MMP2 and 9, which are highly abundant in wound tissues, potently degrade gelatin and can be reactivated after treatment with sodium dodecyl sulfate. Since these proteases are visible on zymogram gels in both their mature and pro-forms, gelatin zymography only allows monitoring the amount of potentially active MMP2 and 9 in the sample but provides additional quantitative information on the zymogens and the activated enzymes. Alternatively, fluids were incubated with radioactive collagen as test substrate whose proteolytic processing was followed by autoradiography with the advantage that binding of endogenous inhibitors like TIMPs to the protease is preserved during substrate incubation. Furthermore, zymography can be applied on tissue sections in form of in situ zymography providing additional spatial information on protease activity as demonstrated by analysis of plasmin in human skin wounds using casein as an artificial substrate. Still, while powerful for the analysis of a small subset of wound proteases, zymographic techniques fail to examine the majority of active proteolytic enzymes after wounding. Finally, the activity of proteases towards well-defined substrates during wound healing could be visualized by following the lack of substrate processing in protease knockout animals. Examples are the deposition of fibrin in plasminogen deficient mice revealed by immunohistochemistry, the impaired processing of collagen fibers in MMP9 knockouts shown by electron microscopy and the reduced degradation of connective tissue growth factor (CTGF) in mice lacking MMP13 (Toriseva and Kähäri, 2009).

In recent years, a panel of activity-based probes $(\mathrm{ABP})$ for several protease classes was developed (Fonovic and Bogyo, 2007) that have been successfully applied to detect active proteases in tumor samples. To cover more than one protease this approach was extended by use of probe libraries for 
whole protease classes based on their catalytic mechanism that allowed activity profiling of more than 20 metalloproteases in complex samples. Thereby, the extracted active proteases are identified by mass spectrometry-based proteomics, opening up a new field of research termed activity-based protease proteomics that also expands the possibilities to examine the wound activity degradome. When coupled to fluorescent moieties ABPs become effective agents for protease activity imaging in vivo as demonstrated by several studies analyzing cysteine proteases in tumors. Thereby, active proteases can be visualized in the living animal and their tissue distribution subsequently analyzed on tissue sections. Attractive alternatives to the use of fluorophores as imaging tags are radioactive tracers, such as $\left[{ }^{18} \mathrm{~F}\right]$-labeled ABPs that could be much easier translated to the clinics. Such molecules have been developed for MMPs and successfully applied to in vivo imaging of breast cancer in mice (auf dem Keller et al., 2010). One possible application for this kind of protease activity probes in the field of tissue repair might be the detection and in situ characterization of aberrant internal repair processes with help of radioactive tracer ABPs that are directed against disease associated proteases. Because of the lack of knowledge on extended protease substrate specificities, to date only a few highly specific ABPs are available. However, novel powerful proteomics technologies for the high-throughput characterization of protease cleavage sites, such as Proteomic Identification of Cleavage Sites (PICS) (Schilling and Overall, 2008), will allow developing new ABPs with the potential to more comprehensively profile active proteases during wound repair.

\section{Elucidating wound substrate degradomes}

An ultimate goal in the examination of proteolysis in wound healing is the assignment of protein substrates to active proteases, i.e., the determination of the substrate degradome for each individual proteolytic enzyme, at any given time point during the repair process. Through many years of magnificent biochemical research numerous substrates of proteases known to be active in the healing skin wound have been identified, whereby mostly in vitro or cell-based assays were employed. Cleavage of some of these substrates, predominantly ECM proteins, could be further validated in vivo and in some cases directly related to the responsible protease by use of knockout mice or to a protease class by applying inhibitors directly to the wound site (Toriseva and Kähäri, 2009).

With the rapid advances in quantitative proteomics this technology became a powerful tool for the identification of protease substrates that when degraded are substantially reduced in amount in a protease exposed compared to a control sample or enriched in cell culture supernatants by proteolytic shedding from the membrane. However, this approach fails to detect specific cleavages that are responsible for the proteolytic modification of bioactive proteins and thus the modulation of their activity. To overcome this limitation various novel techniques have been developed that enable the selective identification of protein $\mathrm{N}$-termini (N-terminome) and the assignment of substrates and cleavage sites to test proteases (auf dem Keller and Schilling, 2010). This can be achieved by selective labeling of N-terminal protein $\alpha$-amines using biotinylated reagents and subsequent pullout of $\mathrm{N}$-terminal peptides by avidin affinity resins following tryptic digest of the sample. Thereby, labels are transferred after selective blocking of $\varepsilon$-amines in lysine side chains ( $\mathrm{N}$-terminomics) or by use of a modified enzyme that specifically attacks unprotected protein $\mathrm{N}$-termini. As an alternative, all primary amines on a protein are labeled, the sample trypsinated and $\mathrm{N}$-terminal peptides negatively enriched after removal of internal tryptic peptides either by sequential chromatography steps (combined fractional diagonal chromatography, COFRADIC) or by an amine reactive polymer in a recently developed method termed terminal amine isotopic labeling of substrates (TAILS) (Kleifeld et al., 2010). The use of isotopic tags in TAILS allows differential labeling of protease exposed and control samples and thereby discriminating $\mathrm{N}$-termini derived from activity of the test protease from those generated by basal proteolysis in native samples based on their quantitative ratios. Furthermore, the modification of TAILS as an ITRAQ (isobaric tags for relative and absolute quantitation)-based method makes it a robust platform for the simultaneous quantitative assessment of $\mathrm{N}$-terminomes in up to eight conditions (Prudova et al., 2010). This is of particular importance when studying time-resolved processes, such as cutaneous wound healing.

By including knockout animals in a murine wound healing model and specifically analyzing time points with known high expression of the protease under study, TAILS or comparable techniques should be suitable to identify physiological substrates of the test protease in vivo. However, the overwhelming complexity of the wound proteome and the often restricted activity of proteases to only small populations of cells might prevent the detection of these specific cleavages. Furthermore, the lack of a single protease in knockout models might lead to perturbations of the proteolytic network resulting in altered protease activities and substrate availabilities that are only secondarily related to the initial cleavages exerted by the test protease (Kruger, 2009). As a consequence directly monitoring the differential processing of proteins in wild-type and knockout mice could reveal changes in substrate patterns that do not necessarily allow conclusions on the primary substrates of the protease for that corresponding DNA sequences had been deleted from the genome. Hence, it will be crucial to first identify these substrates in vitro and then to specifically analyze their cleavage in vivo, while concomitantly monitoring the general consequences of lack of the responsible protease on proteolytic activities in the tissue. Therefore, we propose an integrated bottom-up and top-down strategy for the identification of physiological substrates of a test protease, the analysis of its influence on the proteolytic network and the elucidation of novel proteases and their activities implicated in cutaneous tissue repair (Figure 1). Thereby, secretomes or cell lysates ideally obtained from primary cells deficient for the test protease that highly express this enzyme in wild-type wounds 


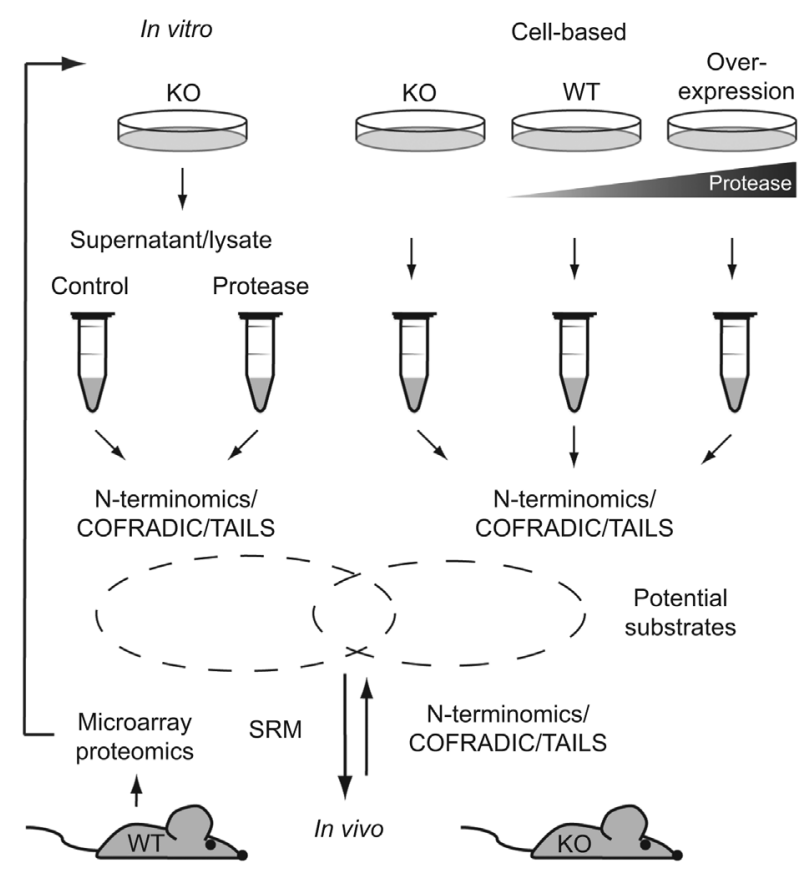

Figure 1 Strategy to elucidate physiological substrates for a test protease, its influence on the protease web and novel proteases and their activities in cutaneous wound healing.

Data from in vitro and cell-based experiments are integrated to develop targeted SRM assays for multiple candidate substrates that are monitored in an in vivo wound healing model. Unbiased $\mathrm{N}$ terminome analyses in combination with microarrays and quantitative proteomics define novel proteases that are subjected to substrate degradomics.

KO, knockout; WT, wild-type; SRM, selected reaction monitoring; COFRADIC, combined fractional diagonal chromatography; TAILS, terminal amine isotopic labeling of substrates.

are first in vitro incubated with the active recombinant protein or control and subjected to differential analysis of their $\mathrm{N}$-terminomes. In these experiments inhibitors for proteases other than the protease under study could be included to more efficiently discriminate direct targets from proteins cleaved by proteolytic enzymes activated by the test protease in the sample. This defines a maximal number of potential substrates that is narrowed down by complementary experiments, where N-terminomes from wild-type cells are compared to those from knockouts and/or cells overexpressing the protease of interest. Additionally, information from the in vitro experiment is used to define if a substrate candidate that can only be assigned with a high false discovery rate in this more physiological cell-based setup is included in further analyses. Both datasets are then combined to define a set of candidate substrates, for which specific SRM assays are designed to study their processing in vivo in the presence and absence of the test protease by specifically monitoring the cleavage site. The integration of these results with data obtained from unbiased multiplex N-terminome analyses of samples from wild-type and knockout animals finally determines parameters for novel substrate candidates that can only be observed in vivo. Furthermore, these data reveal pertur- bations to the protease web during wound repair that in combination with microarray and quantitative proteomics analyses identify novel proteases that are analyzed for their substrate degradomes as described above.

\section{Conclusions}

A tremendous body of work has established proteases as pivotal players in all phases of wound repair. Thereby, they have been identified as important modifiers of the extracellular matrix in reepithelialization and remodeling and more recently also as modulators of multiple signaling pathways by specific processing of bioactive proteins. Most importantly, aberrant proteolytic activity has been strongly associated with the pathogenesis of chronic wound healing that affects the quality of life of many individuals and puts a significant burden on the health systems. Since proteases do not act alone but form a complex interaction network, it will be crucial to systematically determine their expression, activity and substrates at multiple time points of the repair process. Thereby, novel system-wide approaches as described in this minireview open up new possibilities to address this challenging task and have the potential to identify new targets to therapeutically interfere with causes for aberrant skin repair.

\section{Acknowledgments}

U.a.d.K. acknowledges the support by a Marie Curie International Reintegration Grant (FP7-PEOPLE-2010-RG) from the European Commission.

\section{References}

auf dem Keller, U., Bellac, C.L., Li, Y., Lou, Y., Lange, P.F., Ting, R., Harwig, C., Kappelhoff, R., Dedhar, S., Adam, M.J., et al. (2010). Novel matrix metalloproteinase inhibitor $\left[{ }^{18} \mathrm{~F}\right]$ marimastataryltrifluoroborate as a probe for in vivo positron emission tomography imaging in cancer. Cancer Res. 70, 7562-7569.

auf dem Keller, U., Doucet, A., and Overall, C.M. (2007). Protease research in the era of systems biology. Biol. Chem. 388, 11591162 .

auf dem Keller, U. and Schilling, O. (2010). Proteomic techniques and activity-based probes for the system-wide study of proteolysis. Biochimie 92, 1705-1714.

Caldwell, R.L., Opalenik, S.R., Davidson, J.M., Caprioli, R.M., and Nanney, L.B. (2008). Tissue profiling MALDI mass spectrometry reveals prominent calcium-binding proteins in the proteome of regenerative MRL mouse wounds. Wound Repair Regen. 16, 442-449.

Deonarine, K., Panelli, M.C., Stashower, M.E., Jin, P., Smith, K., Slade, H.B., Norwood, C., Wang, E., Marincola, F.M., and Stroncek, D.F. (2007). Gene expression profiling of cutaneous wound healing. J. Transl. Med. 5, 11.

Edsberg, L.E. (2009). Proteomic Approaches for Studying the Phases of Wound Healing. In: Bioengineering Research of Chronic Wounds. A. Gefen, ed. (Berlin, Heidelberg: Springer), pp. 343362. 
Eming, S.A., Koch, M., Krieger, A., Brachvogel, B., Kreft, S., Bruckner-Tuderman, L., Krieg, T., Shannon, J.D., and Fox, J.W. (2010). Differential proteomic analysis distinguishes tissue repair biomarker signatures in wound exudates obtained from normal healing and chronic wounds. J. Proteome Res. 9, 47584766.

Escalante, T., Rucavado, A., Pinto, A.F.M., Terra, R.M.S., Gutiérrez, J.M., and Fox, J.W. (2009). Wound exudate as a proteomic window to reveal different mechanisms of tissue damage by snake venom toxins. J. Proteome Res. 8, 5120-5131.

Fernandez, M.L., Broadbent, J.A., Shooter, G.K., Malda, J., and Upton, Z. (2008). Development of an enhanced proteomic method to detect prognostic and diagnostic markers of healing in chronic wound fluid. Br. J. Dermatol. 158, 281-290.

Fonovic, M. and Bogyo, M. (2007). Activity based probes for proteases: applications to biomarker discovery, molecular imaging and drug screening. Curr. Pharm. Des. 13, 253-261.

Gurtner, G.C., Werner, S., Barrandon, Y., and Longaker, M.T. (2008). Wound repair and regeneration. Nature 453, 314-321.

Hodgkinson, L.M., Wang, L., Duncan, G., Edwards, D.R., and Wormstone, I.M. (2010). ADAM and ADAMTS gene expression in native and wound healing human lens epithelial cells. Mol. Vis. 16, 2765-2776.

Huttenhain, R., Malmstrom, J., Picotti, P., and Aebersold, R. (2009). Perspectives of targeted mass spectrometry for protein biomarker verification. Curr. Opin. Chem. Biol. 13, 518-525.

Kappelhoff, R., Auf dem Keller, U., and Overall, C.M. (2010). Analysis of the degradome with the CLIP-CHIP microarray. Methods Mol. Biol. 622, 175-193.

Kleifeld, O., Doucet, A., auf dem Keller, U., Prudova, A., Schilling, O., Kainthan, R.K., Starr, A.E., Foster, L.J., Kizhakkedathu, J.N., and Overall, C.M. (2010). Isotopic labeling of terminal amines in complex samples identifies protein $\mathrm{N}$-termini and protease cleavage products. Nat. Biotechnol. 28, 281-288.

Kruger, A. (2009). Functional genetic mouse models: promising tools for investigation of the proteolytic internet. Biol. Chem. 390, 91-97.

Madlener, M., Parks, W.C., and Werner, S. (1998). Matrix metalloproteinases (MMPs) and their physiological inhibitors (TIMPs) are differentially expressed during excisional skin wound repair. Exp. Cell Res. 242, 201-210.

Moali, C. and Hulmes, D.J.S. (2009). Extracellular and cell surface proteases in wound healing: new players are still emerging. Eur. J. Dermatol. 19, 552-564.

Nanney, L.B., Caldwell, R.L., Pollins, A.C., Cardwell, N.L., Opalenik, S.R., and Davidson, J.M. (2006). Novel approaches for understanding the mechanisms of wound repair. J. Investig. Dermatol. Symp. Proc. 11, 132-139.

Page-McCaw, A., Ewald, A.J., and Werb, Z. (2007). Matrix metalloproteinases and the regulation of tissue remodelling. Nat. Rev. Mol. Cell Biol. 8, 221-233.

Pennington, C.J. and Edwards, D.R. (2010). Real-time PCR expression profiling of MMPs and TIMPs. Methods Mol. Biol. 622, 159-173.

Picotti, P., Lam, H., Campbell, D., Deutsch, E.W., Mirzaei, H., Ranish, J., Domon, B., and Aebersold, R. (2008). A database of mass spectrometric assays for the yeast proteome. Nat. Methods 5, 913-914.

Prudova, A., auf dem Keller, U., Butler, G.S., and Overall, C.M. (2010). Multiplex N-terminome analysis of MMP-2 and MMP9 substrate degradomes by iTRAQ-TAILS quantitative proteomics. Mol. Cell. Proteomics 9, 894-911.

Rittié, L., Perbal, B., Castellot, J.J., Orringer, J.S., Voorhees, J.J., and Fisher, G.J. (2011). Spatial-temporal modulation of CCN proteins during wound healing in human skin in vivo. J. Cell Commun. Signal. 5, 69-80.

Roy, S., Patel, D., Khanna, S., Gordillo, G.M., Biswas, S., Friedman, A., and Sen, C.K. (2007). Transcriptome-wide analysis of blood vessels laser captured from human skin and chronic wound-edge tissue. Proc. Natl. Acad. Sci. USA 104, 14472-14477.

Rucavado, A., Escalante, T., Shannon, J., Gutierrez, J.M., and Fox, J.W. (2011). Proteomics of wound exudate in snake venominduced pathology: search for biomarkers to assess tissue damage and therapeutic success. J. Proteome Res. 10, 1987-2005.

Schilling, O. and Overall, C.M. (2008). Proteome-derived, databasesearchable peptide libraries for identifying protease cleavage sites. Nat. Biotechnol. 26, 685-694.

Schwartz, D.R., Moin, K., Yao, B., Matrisian, L.M., Coussens, L.M., Bugge, T.H., Fingleton, B., Acuff, H.B., Sinnamon, M., Nassar, H., et al. (2007). Hu/Mu ProtIn oligonucleotide microarray: dual-species array for profiling protease and protease inhibitor gene expression in tumors and their microenvironment. Mol. Cancer Res. 5, 443-454.

Toriseva, M. and Kähäri, V.-M. (2009). Proteinases in cutaneous wound healing. Cell. Mol. Life Sci. 66, 203-224.

Wisniewski, J.R., Ostasiewicz, P., and Mann, M. (2011). High recovery FASP applied to the proteomic analysis of microdissected formalin fixed paraffin embedded cancer tissues retrieves known colon cancer markers. J. Proteome Res. 10, 3040-3049.

Received May 29, 2011; accepted July 6, 2011; previously published online August 6, 2011 